\title{
Microplane Model M5f for Multiaxial Behavior and Fracture of Fiber-Reinforced Concrete
}

\author{
Alessandro Beghini'; Zdeněk P. Bažant, F.ASCE²; Yong Zhou ${ }^{3}$; Olivier Gouirand ${ }^{4}$; and Ferhun C. Caner ${ }^{5}$
}

\begin{abstract}
Despite impressive advances, the existing constitutive and fracture models for fiber-reinforced concrete (FRC) are essentially limited to uniaxial loading. The microplane modeling approach, which has already been successful for concrete, rock, clay, sand, and foam, is shown capable of describing the nonlinear hardening-softening behavior and fracturing of FRC under not only uniaxial but also general multiaxial loading. The present work generalizes model M5 for concrete without fibers, the distinguishing feature of which is a series coupling of kinematically and statically constrained microplane systems. This feature allows simulating the evolution of dense narrow cracks of many orientations into wide cracks of one distinct orientation. The crack opening on a statically constrained microplane is used to determine the resistance of fibers normal to the microplane. An effective iterative algorithm suitable for each loading step of finite element analysis is developed, and a simple sequential procedure for identifying the model parameters from test data is formulated. The model allows a close match of published test data on uniaxial and multiaxial stress-strain curves, and on multiaxial failure envelopes.
\end{abstract}

DOI: 10.1061/(ASCE)0733-9399(2007)133:1(66)

CE Database subject headings: Fracture; Concrete, reinforced; Stress strain relations; Numerical models.

\section{Introduction}

The use of fibers to strengthen brittle materials dates back to the Roman times when people mixed straw or horsehair with clay to make better bricks. Fiber-reinforced concrete (FRC) is a continuation of the same idea. Fibers of all orientations, randomly embedded in FRC, serve to restrict and delay the coalescence of microcracks, microvoids, and microslips into wide continuous cracks. While the fibers increase the uniaxial tensile and compressive strength only moderately, they are very effective for enhancing the ductility and energy absorption capability. This elevates structure resistance to impact, groundshock, and blast (important, e.g., as an antiterrorist defense). By limiting crack width, fiber reinforcement counteracts permeability increase due to cracking and thus reduces corrosion risk. Besides, allowing omission of traditional steel reinforcement reduces labor costs.

\footnotetext{
${ }^{1}$ Skidmore, Owings \& Merrill LLP, 224 S. Michigan Ave., Chicago, IL 60604; formerly, Graduate Research Assistant, Northwestern Univ., 2145 Sheridan Rd., CEE, Evanston, IL 60208.

${ }^{2}$ McCormick Institute Professor and W.P. Murphy Professor of Civil Engineering and Materials Science, Northwestern Univ., 2145 Sheridan Rd., CEE, Evanston, IL 60208 (corresponding author).

${ }^{3}$ Graduate Research Assistant, Northwestern Univ., 2145 Sheridan Rd., CEE, Evanston, IL 60208.

${ }^{4}$ Predoctoral Visiting Fellow, Northwestern Univ., 2145 Sheridan Rd., CEE, Evanston, IL 60208; on leave from Ecole Polytechique, France.

${ }^{5}$ Ramón y Cajal Fellow, Technical Univ., of Catalonia (UPC), Barcelona, Spain; formerly, Visiting Scholar, Northwestern Univ., 2145 Sheridan Rd., CEE, Evanston, IL 60208.

Note. Associate Editor: Yunping Xi. Discussion open until June 1, 2007. Separate discussions must be submitted for individual papers. To extend the closing date by one month, a written request must be filed with the ASCE Managing Editor. The manuscript for this paper was submitted for review and possible publication on July 21, 2005; approved on March 3, 2006. This paper is part of the Journal of Engineering Mechanics, Vol. 133, No. 1, January 1, 2007. @ASCE, ISSN 0733-9399/2007/1-66$75 / \$ 25.00$.
}

Most of the previous experiments as well as formulations have been limited to uniaxial tension ( $\mathrm{Li}$ et al. 1998; Nataraja et al. 1999; Ramesh et al. 2003). Such knowledge, however, is insufficient for structural design in general. Recently, though, valuable multiaxial loading experiments have been reported by Yin et al. (1989), Traina and Mansour (1991), Chern et al. (1992), Pantazopoulou and Zanganeh (2001), Kwak et al. (2002), Mirsayah and Banthia (2002), and Cho and Kim (2003).

The experimental investigations have been accompanied by constitutive modeling efforts: Kullaa (1994), Nataraja et al. (1999), Grimaldi and Luciano (2000), Peng and Meyer (2000), Li and Li (2001), Kholmyansky (2002), Kwak et al. (2002), Cho and Kim (2003), Ramesh et al. (2003), and Kabele (2004). Nevertheless, the existing models are still unable to capture realistically the experimental evidence from multiaxial testing. They describe well only specific loading conditions, mostly uniaxial tension (Kullaa 1994; Pizzari et al. 2000; Li and Li 2001) and uniaxial compression (Nataraja et al. 1999; Ramesh et al. 2003). This prevents applications in finite element programs.

The fact that the traditional constitutive models are expressed directly in terms of tensors and their invariants complicates their development. To overcome this difficulty, we adopt the microplane modeling approach in which the constitutive model is defined by relations between stress and strain vectors on a plane of generic orientation. The new model, named M5f, generalizes the recently published microplane Model M5 (Bažant and Caner 2005a,b) for concrete without fibers. The stress-strain boundaries of model M5 are modified by superposing the resistance of rupturing and slipping fibers crossing the cracks.

\section{Background on Microplane Approach and on Models M4 and M5}

Inspired by the slip theory of plasticity of Batdorf and Budiansky (1949), based on Taylor's (1938) idea, Bažant and Oh (1983a, 1985) introduced the microplane model to simulate materials 


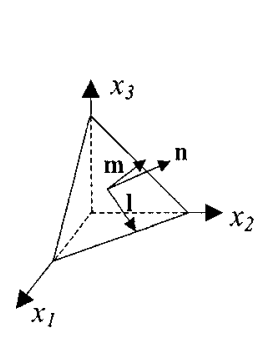

(a)

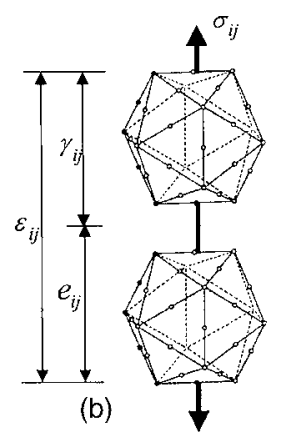

(c)

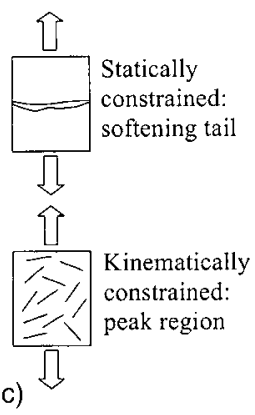

Fig. 1. (a) Local coordinate system on a microplane; (b) coupling of a kinematically and a statically constrained system in microplane Model M5; and (c) main purpose of each microplane system in Model M5

undergoing softening damage. This model, in which the microstructure is constrained not only statically but also kinematically, can capture the fact that most inelastic phenomena in the concrete microstructure, such as crack opening, frictional slip and dilatancy, compressive splitting with lateral spreading, and fiber breaks or pullouts, have distinct spatial orientations which cannot be captured by the classical tensorial constitutive models exemplified by the theories of plasticity. The model rests on two basic ideas: (1) describe these microstructural phenomena by a constitutive relation expressed not in terms of stress and strain tensors of the macroscopic continuum, but in terms of stress and strain vectors acting on planes of all possible orientations at a given point of the continuum; and (2) use a variational principle to relate the microplane vectors (the micro) to the continuum tensors (the macro). Despite increased computer time requirements, this approach has many advantages, described in detail in Bažant et al. (2000a).

Since 1983, the microplane model for concrete has evolved through five progressively improved versions, labeled as M1, M2, M3, M4, and M5 (Bažant and Caner 2005a). Microplane models have also been developed for other complex materials such as rock (Bažant and $\mathrm{Zi} 2003$ ), sand, clay, rigid foam, shape memory alloys, and fiber composites (Brocca and Bažant 2001; Brocca et al. 2001), and have been generalized to finite strain (Bažant et al. 2000b; Carol 2004).

Model M4 (Bažant et al. 2000a; Caner and Bažant 2000) has one limitation-it does not describe well the far postpeak softening. In particular, it does not allow the tensile stress across cracks to be reduced exactly to zero at large crack openings. Thus M4 cannot capture accurately the total energy absorption. This was remedied in Model M5 for concrete (Bažant and Caner 2005a,b) by introducing a series coupling of statically and kinematically constrained microplane models, as shown in Figs. 1(b and c).

\section{Review of Basic Relations in Microplane Model M5}

According to the series coupling assumption in Model M5 (Bažant and Caner 2005a,b), the stress tensor $\sigma_{i j}$ is the same in the statically and kinematically constrained parts, and the strain tensor $\epsilon_{i j}$ of the macroscopic continuum is decomposed as $\epsilon_{i j}=\gamma_{i j}+e_{i j}$, where indices $i, j=1,2,3$ refer to Cartesian coordinates; and $\gamma_{i j}$ or $e_{i j}=$ strain in the statically or kinematically constrained microplane system [see Fig. 1(b)]. In the computational algorithm of Model M5 (Bažant and Caner 2005a,b), the strain tensors in the statically and kinematically constrained parts are iteratively adjusted to minimize the stress tensor differences to almost zero.

In the statically constrained system, which simulates the softening due to cohesive crack opening, the macrostress is projected onto each microplane as $\sigma_{N}=N_{i j} \sigma_{i j}, \sigma_{L}=L_{i j} \sigma_{i j}, \sigma_{M}=M_{i j} \sigma_{i j}$ where $N_{i j}=n_{i} n_{j}, L_{i j}=\left(l_{i} n_{j}+l_{j} n_{i}\right) / 2$, and $M_{i j}=\left(m_{i} n_{j}+m_{j} n_{i}\right) / 2$; $n_{i}=$ normal vector on the microplane; $m_{i}$ and $l_{i}$ are orthogonal to $n_{i}$ [see Fig. 1(a)]; and repeated indices imply summation. Ideally, there should be infinitely many microplanes with all spatial orientations, but for numerical integration a finite number of microplanes must be used. Optimally, the microplane normals match the points of a Gaussian integration formula for the surface of a sphere. Such integration formulas were extensively investigated in mathematics (Stroud 1971) and some improved formulas were contributed by Bažant and Oh (1986). The stress vectors are then used in the softening law of a vectorial cohesive crack model to obtain the corresponding strain vectors $\gamma_{N}, \gamma_{M}$, and $\gamma_{L}$. Finally, the continuum strain tensor is obtained according to the principle of complementary virtual work

$$
\gamma_{i j}=\frac{3}{2 \pi} \int_{\Omega} u_{i j} \mathrm{~d} \Omega \simeq 6 \sum_{\mu=1}^{N_{m}} w_{\mu} u_{i j}^{(\mu)}
$$

where $u_{i j}=\gamma_{N} N_{i j}+\gamma_{M} M_{i j}+\gamma_{L} L_{i j} ; \Omega=$ surface of a unit hemisphere; $N_{m}=$ number of microplanes considered; and $w_{\mu}=$ weight of each microplane, i.e., the weight of each integration point in the numerical integration formula for a spherical surface (Bažant and Oh 1986).

In the kinematically constrained system, the approach is opposite- the vectorial constitutive law is used to derive the stress from strain. First the continuum strain tensor $e_{i j}$ is projected onto each microplane: $e_{N}=N_{i j} e_{i j}, e_{L}=L_{i j} e_{i j}, e_{M}=M_{i j} e_{i j}$. Same as in M4 (Bažant et al. 2000a), a volumetric-deviatoric split must then be applied to each normal component as $e_{D}=e_{N}-e_{V}$ with $e_{V}=$ volumetric strain and $e_{D}=$ deviatoric component of the normal microplane strain. The volumetric component is the same for all the microplanes and equal to the macroscopic volumetric strain. Then, $e_{V}, e_{D}, e_{N}, e_{M}$, and $e_{L}$ are used in the vectorial constitutive law to obtain the components $s_{V}, s_{D}, s_{N}, s_{M}$, and $s_{L}$ of the microplane stress vector. Finally, the continuum macrostress tensor is obtained from the principle of virtual work

$$
\sigma_{i j}=s_{V} \delta_{i j}+s_{i j}^{D} \quad s_{i j}^{D}=\frac{3}{2 \pi} \int_{\Omega} s_{i j} \mathrm{~d} \Omega \simeq 6 \sum_{\mu=1}^{N_{m}} w_{\mu} s_{i j}^{(\mu)}
$$

where $s_{i j}=s_{D}\left(N_{i j}-\delta_{i j} / 3\right)+s_{M} M_{i j}+s_{L} L_{i j}$; and the formula for optimal Gaussian numerical integration is the same as in Eq. (1).

\section{Microplane Constitutive Laws and Their Physical Basis}

Capturing the far-off postpeak tensile softening and total energy absorption in dynamically loaded structures was the objective in developing microplane Model M5. This objective is even more important for FRC because large crack openings are needed to activate the resistance of fibers, the energy dissipation of which must be captured correctly. In what follows, the resistance of fibers and their interaction with concrete will be incorporated into the boundary curves of the original Model M5. This needs to be done for both the statically and kinematically constrained microplane systems. 

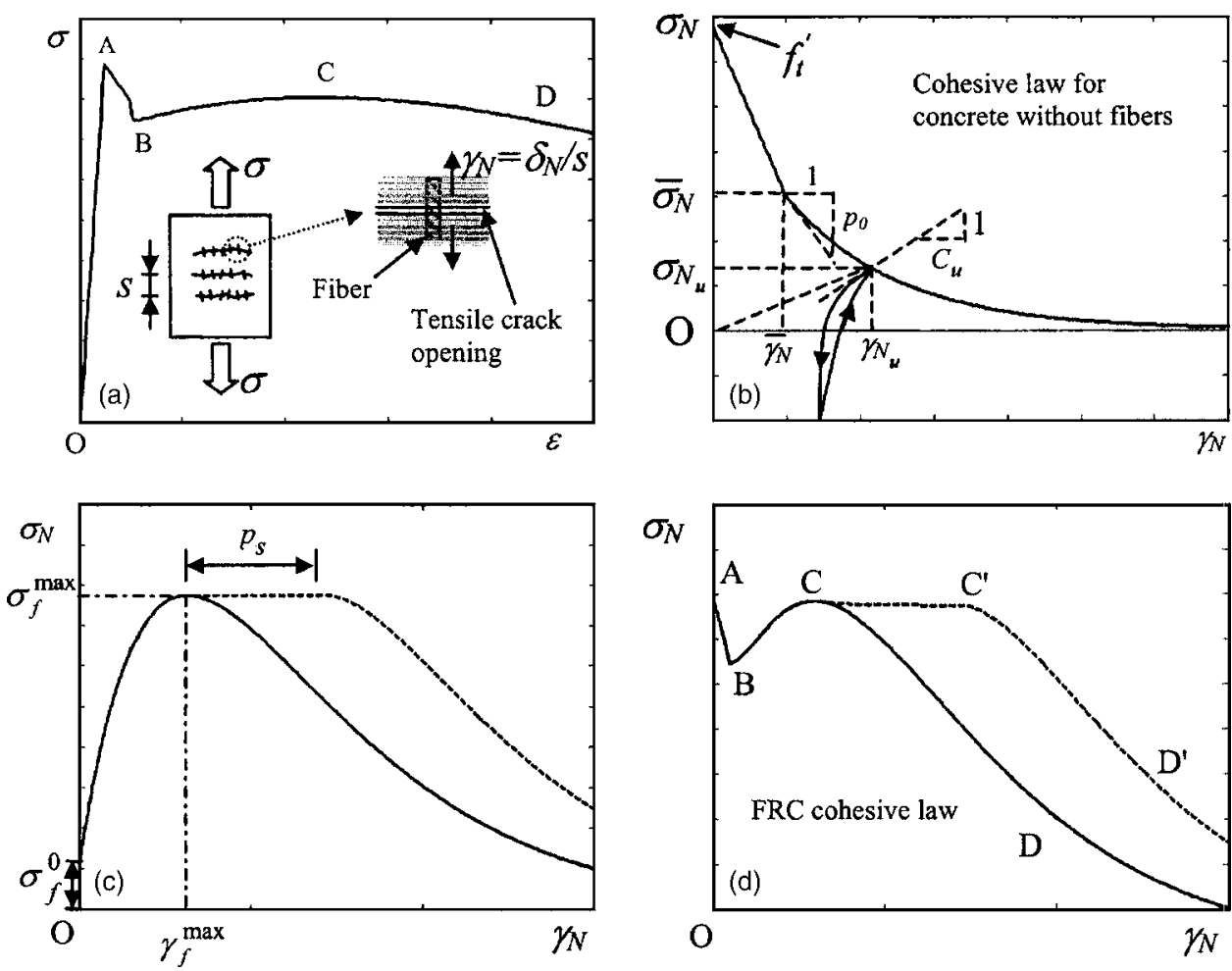

Fig. 2. (a) Typical experimental behavior observed in uniaxial tension for FRC; (b) cohesive law for concrete without fibers ( $p_{0}$ and $C_{u}$ stand for $p_{0} \bar{\sigma}_{N} / \bar{\gamma}_{N}$ and $C_{u} \gamma_{N_{0}} / \gamma_{N_{0}}$ ); (c) contribution of fibers to crack-bridging stress; (d) overall cohesive response of FRC on the microplane

\section{Statically Constrained System: Cohesive Cracks and Fiber Resistance}

The constitutive law for the statically constrained microplanes in M5 is based on the cohesive crack model, which is active only in the postpeak tensile softening. The cohesive crack model for concrete must be enhanced by a simple model for the resistance of fibers crossing the crack. The typical response of FRC in uniaxial tension is shown in Fig. 2(a). Compared to normal concrete, the presence of fibers randomly distributed increases the resistance to tensile fracture of the resulting composite, as sketched in the inset of Fig. 2(a). This additional resistance due to fibers is taken into account by assuming that, at the microplane level, the concrete matrix and the fibers are subjected to the same strain $\gamma_{N}$ in every direction. In other words, the fibers and concrete (distinguished by superscripts $f$ and $c$ ) are assumed to work in parallel coupling, and so their microplane stress vectors are simply summed: $\sigma_{i}=\sigma_{i}^{f}+\sigma_{i}^{c}(i=1,2,3)$. For a continuum fracture description, $\gamma=\delta / h=$ smeared cracking strain, where $\delta=$ crack opening and $h$ represents either the width of one finite element (in the crack band model), or the width of the nonlocal averaging zone (in a nonlocal model), or the minimum possible mean spacing of parallel cracks (if they do not localize) (Bažant 1985; Bažant and Jirásek 2002; Bažant and Pijaudier-Cabot 1989).

Mixed-mode cohesive fracture is considered in Model M5 (Bažant and Caner 2005a,b) through a mode mixity parameter $\beta=$ ratio between shear and tensile components of relative displacement between crack faces [as introduced by Camacho and Ortiz (1996); see also Bažant and Caner (2005a,b)].

Here, for simplicity, the resistance of cracks to shear is not included, with two justifications: (1) no relevant test data exist; and (2) the shearing of cracks seems less important since fibers resist mainly crack opening. Thus, in M5f, the statically constrained microplanes represent only Mode I crack opening, and so the general relation $\sigma_{i}=\sigma_{i}^{f}+\sigma_{i}^{c}$ reduces to $\sigma_{N}=\sigma_{N}^{f}+\sigma_{N}^{c}$.

The strain $\gamma_{i j}$ is zero until one of the microplanes reaches the tensile strength, at which point a cohesive crack forms on that microplane. For the softening law on the microplane, scaled by the factor $h$, we assume the tensile cohesive (crack-bridging) normal stress $\sigma_{N}^{c}$ to start its descent as a linear function of $\gamma=\delta_{N} / h$ [Fig. 2(b)] and then decay exponentially

$$
\begin{gathered}
\sigma_{N}^{c}=\left(\bar{\sigma}_{N}-f_{t}^{\prime}\right) \gamma_{N} / \bar{\gamma}_{N}+f_{t}^{\prime} \quad\left(\gamma_{N} \leq \bar{\gamma}_{N}\right), \\
\sigma_{N}^{c}=\bar{\sigma}_{N} \mathrm{e}^{-p 0\left(\gamma_{N} / \bar{\gamma}_{N}-1\right)} \quad\left(\gamma_{N}>\bar{\gamma}_{N}\right)
\end{gathered}
$$

where $f_{t}^{\prime}=$ tensile strength of concrete; $\bar{\gamma}_{N}$ and $\bar{\sigma}_{N}=$ coordinates of the transition point; and $p_{0}$ controls the initial slope of exponential decay. If the bilinear softening law from the original formulation of M5 were applied to FRC, the convergence would be slower for states near the point of slope change in the softening law. The smooth softening law in Eq. (3) gives faster convergence.

The fiber contribution to the bridging stress $\sigma_{N}^{f}$ is represented by another cohesive law, scaled again by factor $h$. We adopt a simplified form of Kholmyansky's (2002) equation, shown as the continuous line in Fig. 2(c)

$$
\sigma_{N}^{f}=p_{1}\left\langle\gamma_{N}+\sigma_{f}^{0} / p_{1}\right\rangle \mathrm{e}^{-p_{2} \gamma_{N}} \quad\left(\gamma_{N}>0\right)
$$

where $p_{1}, p_{2}$, and $\sigma_{f}^{0}=$ empirical parameters depending on volume fraction $V_{f}$ of fibers and on their geometric characteristics; and $\langle X\rangle=\max (X, 0)$ (Macauley brackets). Same as microplane Models M3 (Bažant et al. 1996), M4, and M5, the inelastic behavior is modeled by imposing, in the microplane stress-strain space, stress-strain boundaries, representing strain-dependent yield 

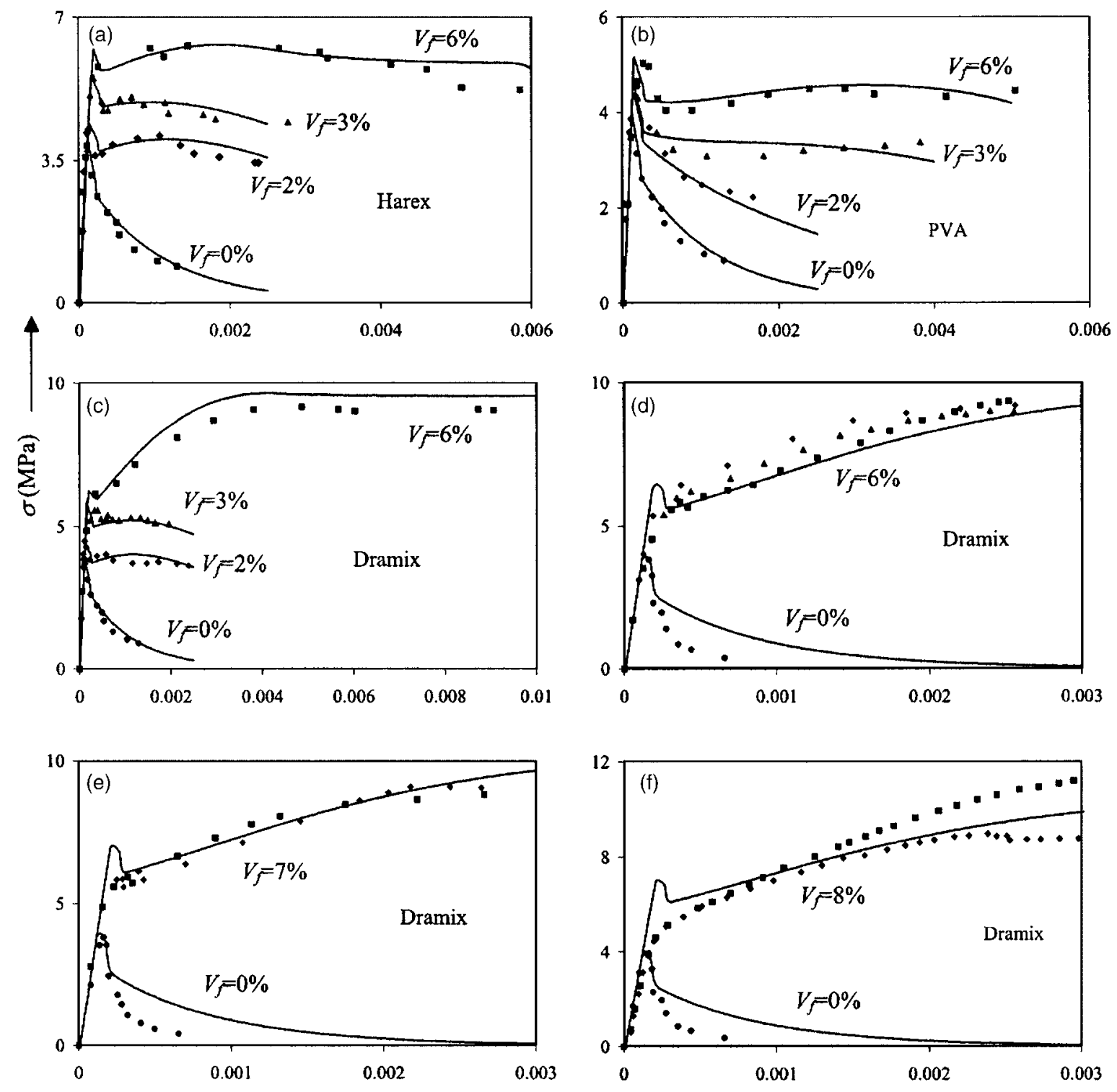

Fig. 3. Experimental data and numerical simulation for uniaxial tension

limits that can never be exceeded. Within these boundaries, all microplane loading, unloading, and reloading is assumed to be elastic.

The overall cohesive stress curve for the microplane is obtained as the sum of the cohesive softening law for concrete, given by Eq. (3), and of the fiber resistance law (4); see line ABCD in Fig. 2(d). This cohesive curve can be directly related to the macroscopic behavior experimentally observed in uniaxial tension tests of FRC, as sketched in Fig. 2(a) (line ABCD). The FRC specimen behaves almost elastically up to the peak [point A in Fig. 2(d)], which agrees with the fact that the statically constrained microplane system remains inactive (i.e., rigid) until the tensile strength of the FRC is first reached on one microplane. Then the cracks normal to the load start to open and cause softening (line AB), while the crack opening still remains small. At a certain point on the softening curve (point B), the crack opening triggers fiber resistance, which causes the material to reharden up to a second peak (point $\mathrm{C}$ ). The final softening (curve CD) is controlled by development of debonding cracks, pullout of fibers from the crack faces, and fiber ruptures. The parameters in Eq. (4) reflect the individual features of this behavior as shown in Fig. 2(d); $\sigma_{f}^{0}$ gives the contribution of fibers to the first stress peak (point $\mathrm{A}$ ), while parameters $p_{1}$ and $p_{2}$ mainly control the location of the second peak (point $\mathrm{C}$ ).

The cohesive curve must obviously depend also on the type and geometry of the fibers. For FRC with a high $V_{f}$ content $\left(V_{f} \geq 0.06\right)$ of fibers with hooks at ends (marketed as Harex and Dramix), Figs. 3(a and c) show a variant of Eq. (4), in which the onset of softening is preceded by a long horizontal yield plateau (of length $p_{s}$ ) reflecting the resistance of fibers to crack openings before the final stage of fiber pullout and rupture. The plateau, which follows the prepeak expression (4), is represented by the dashed line in Fig. 2(c). The resulting overall cohesive curve is shown in Fig. 2(d) by the dashed line $\mathrm{ABC}^{\prime} \mathrm{D}^{\prime}$.

The unloading and reloading of a cohesive crack in concrete without fibers may be described by 

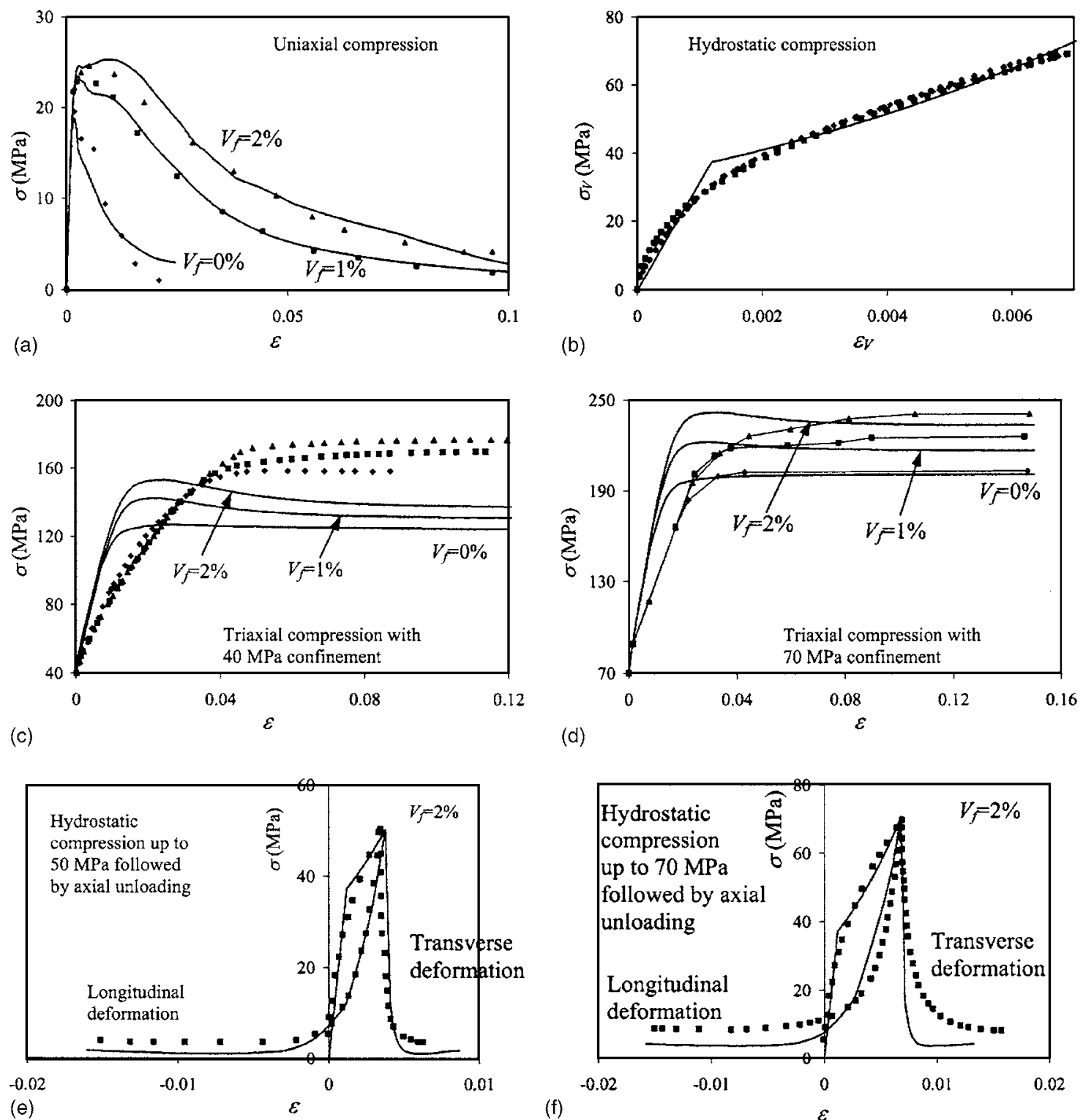

(d)

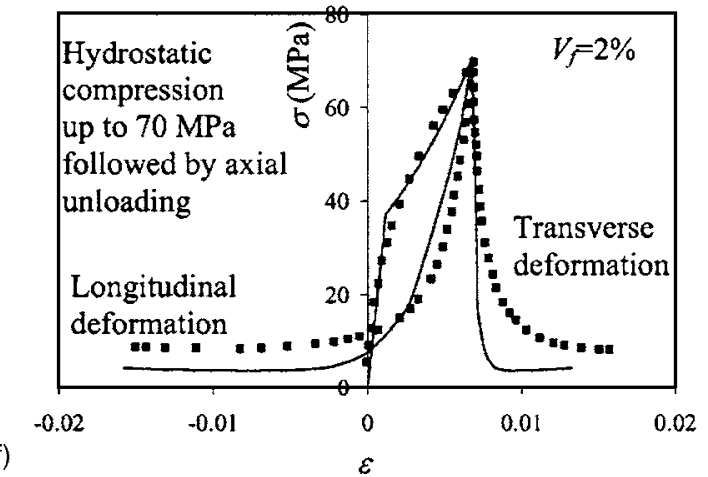

Fig. 4. Experimental data and numerical simulation for multiaxial loading

$$
\gamma_{N}=\gamma_{N_{u}}+C_{u}\left(\sigma_{N}\right) \gamma_{N_{u}} \Delta \sigma_{N} / \sigma_{N_{u}}
$$

[Fig. 2(b)] where $C_{u}\left(0<C_{u}<1\right)$ characterizes the degree of reversibility of the crack opening; and $\gamma_{N_{u}}$ and $\sigma_{N_{u}}=$ maximum strain and maximum stress attained in the computation. $C_{u}=1$ gives reversible and $C_{u}=0$ irreversible opening, but in reality the crack opening is only partially reversible, because of debris deposited between the crack faces [see experiments by Reinhardt and Cornelissen (1984)]. Therefore, $C_{u}$ must decay, and here an exponential decay is assumed [Fig. 2(b)] in the form $C_{u}=p_{u} \mathrm{e}^{\sigma_{N}-\sigma_{N_{u}}}$, where $p_{u}=$ parameter controlling the initial slope of the unloading curve $\left(0 \leq p_{u} \leq 1\right)$. In general, unloading and reloading can be characterized by different $p_{u}$ [see Fig. 2(b)].

The considerations for concrete without fibers may be extended to the overall unloading-reloading characteristics of FRC. Unfortunately, there seem to exist no data for calibrating parameter $p_{u}$ for FRC, and so the mean of 0.5 is suggested as a default value. Nevertheless, the uncertainty about $C_{u}$ is not a serious impediment because unloading is governed mainly by the kinematically constrained system.

\section{Kinematically Constrained System: Fiber Effect on Its Boundaries}

The kinematically constrained microplane system characterizes the elastic behavior of Model M5f and all the inelastic behaviors other than tensile fracture, especially uniaxial and multiaxial compression. The computational algorithm for the microplane constitutive laws is similar to the classical return mapping algorithm and consists of three basic steps: (1) calculate an elastic predictor; (2) check the stress-strain boundary; and (3) if the boundary is exceeded, return at constant strain onto the boundary. In the case of frictional boundary, which is defined in the plane of normal and shear stress rather than the stress-strain space, the shear stress is returned to the boundary at constant normal stress and constant strains.

The elastic microstress predictor is defined as $\Delta s_{V}=E_{V} \Delta e_{V}$, $\Delta s_{D}=E_{D} \Delta e_{D}, \quad \Delta s_{M}=E_{T} \Delta e_{M}, \Delta s_{L}=E_{T} \Delta e_{L}$, and $\Delta s_{N}=\Delta s_{V}+\Delta s_{D}$ where $\Delta$ denotes the increment in a loading step. The microplane elastic moduli are determined from Young's modulus $E$ and Poisson's ratio $v$ of concrete as $E_{V}=E /(1-2 v), E_{D}=E_{T}=E /(1+\nu)$ 

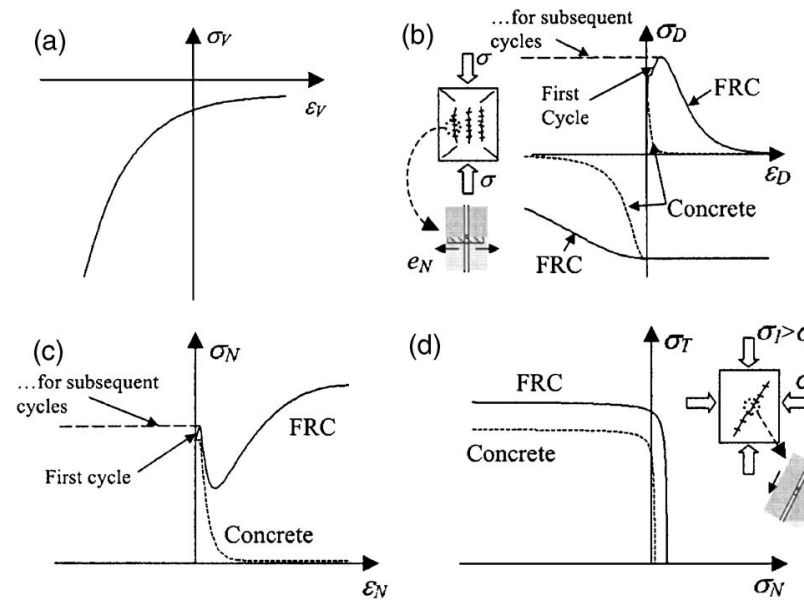

(d)

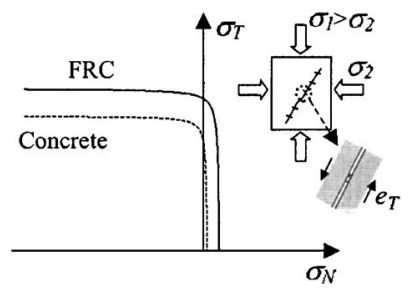

Fig. 5. Boundary surfaces for kinematically constrained model: (a) volumetric boundary; (b) fiber resistance mechanism in uniaxial compression (left) and deviatoric boundary (right); (c) normal boundary; (d) shear boundary (left) and fiber resistance mechanism in triaxial compression (right)

[see Bažant and Prat (1988); Carol and Bažant (1997)]. The elastically incremented total stress must be checked against the stress-strain boundary, i.e.,

$$
s_{V}^{b-} \leq s_{V} \quad s_{D}^{b-} \leq s_{D} \leq s_{D}^{b+} \quad s_{N} \leq s_{N}^{b+} \quad s_{T}=\sqrt{s_{M}^{2}+s_{L}^{2}} \leq s_{T}^{b}
$$

If the stress exceeds the boundary, the new stress value is obtained by returning at constant strain on the boundary.

The stress-strain boundaries of Model M5 need to be modified by superposing the effect of fibers. This is done as follows:

- Compressive volumetric boundary $s_{V}^{b}$. Test data on FRC [Fig. 4(b)] show that the presence of fibers does not affect the response to hydrostatic pressure (at least for $V_{f} \leq 2 \%$ ). Since this loading condition is governed by the compressive volumetric boundary, we conclude that the boundary $s_{V}^{b-}$ needs no adjustment for the effect of fibers. So the boundary used in M5 (which is the same as in M4) is retained [Fig. 5(a)].

- Tensile deviatoric boundary $s_{D}^{b+}$. The shape of the deviatoric boundaries is best inferred from the uniaxial compressive tests. Compared to normal concrete, FRC with high $V_{f}$ has been shown to exhibit only slightly higher strength but much higher energy absorption [see the test data in Fig. 4(a)]. This may be attributed mainly to the resistance of fibers to compressive splitting cracks, as illustrated in Fig. 5(b) on the left. Therefore, the tensile deviatoric boundary is modified similar to the tensile cohesive crack boundary, by assuming a parallel coupling of concrete and fiber effect at the microplane level. So, the additional resistance posed by fibers, expressed as

$$
s_{D f}^{b+}=p_{5}\left\langle e_{D}\right\rangle \mathrm{e}^{-p_{6}\left\langle e_{D}\right\rangle}
$$

must be superposed on the boundary from Model M5 or M4. The shape of the overall boundary $s_{D}^{b+}$ obtained by this superposition is shown in Fig. 5(b) (on the right, by a continuous line). The horizontal boundary is extended into the second quadrant, where it may be reached in cyclic loading.

- Compressive deviatoric boundary $s_{D}^{b-}$. This boundary has the same general shape as in Model M5, however, to capture the experimentally observed postpeak softening in uniaxial compression, a slower decay of the exponential tail of the boundary needs to be introduced. Multiplying coefficient $c_{7}$ of
Model M4 (Caner and Bažant et al. 2000) or M5 by factor 6.5 suffices to match the measured postpeak softening. Also, parameter $c_{8}$, which governs the yielding compressive deviatoric stress and equals 8.0 in M4 and M5, has been decreased to 4.9, to improve the fit. The boundaries $s_{D}^{b-}$ for concrete and for FRC are compared in Fig. 5(b) on the right.

- Tensile normal boundary $s_{N}^{b+}$. It may seem redundant to include this boundary in the kinematically constrained system since the tensile properties are mainly controlled by the statically constrained system through the cohesive crack boundary augmented by the resistance of fibers. However, the tensile normal boundary is needed to prevent excessively large lateral expansion in uniaxial compression. During uniaxial tension, this boundary is usually inactive because the loading occurs in the statically constrained system and the kinematically constrained system is unloading without ever reaching the boundary characterizing concrete without fibers. However, fiber type matters (Soroushian and Bayasi 1991), and for hooked fibers (e.g., Dramix) and high $V_{f}$ [Figs. 3(c-f)], this boundary is reached in computations, due to high elastic stresses. So, in that case, a modification similar to that introduced for $s_{D}^{b+}$ is required to include the fiber effect

$$
s_{N f}^{b+}=p_{3}\left\langle e_{N}\right\rangle \mathrm{e}^{-p_{4}\left\langle e_{N}\right\rangle}
$$

The shape of the overall boundary, resulting as a sum of the concrete boundary (same as in M4 or M5) and additional fiber resistance, is depicted in Fig. 5(c). The horizontal boundary in the second quadrant is needed for cyclic loading.

- Shear boundary $s_{T}^{b}$. It is represented by the same function as in M5. The effect of additional fiber resistance is included in the model by shifting the horizontal asymptote upward and by increasing the value of the cohesion [see Fig. 5(d)]. To effect this shift, M4 parameters $k_{2}$ and $c_{11}$ are made to increase as monotonic functions of $V_{f}$. However, for the loading conditions considered here, parameter $c_{11}$ has no effect. Therefore, it is fixed for all computations as $c_{11}=0.2$, which is the M4 value.

\section{Computational Algorithm}

The microplane Model M5f is meant to be used as a material subroutine in finite element programs, implicit or explicit. In each loading step, in each integration point of each finite element, the finite element program calls this subroutine, which returns the stress tensor for a given strain. The stress tensors for all the points are then used to integrate the internal force vector of the element, which is then assembled to give the internal force vector of the structure.

The main objective, similar to that for Model M5, is to guarantee correct distribution of strain between the statically and kinematically constrained systems, even for large loading steps. This is achieved by solving, at each loading step, the compatibility constraint

$$
r_{i j}=\Delta \epsilon_{i j}-\Delta \gamma_{i j}-\Delta e_{i j}=0
$$

where $\Delta \epsilon_{i j}=$ given strain increment that must be distributed between $\Delta \gamma_{i j}$ (for the statically constrained system) and $\Delta e_{i j}$ (for the kinematically constrained system). Eq. (9) is solved for $\Delta e_{i j}$ by Newton-Raphson iteration based on the tangential fracturing compliance tensor evaluated from the last converged step (with convergence criterion in the form of a very small tolerance for the 

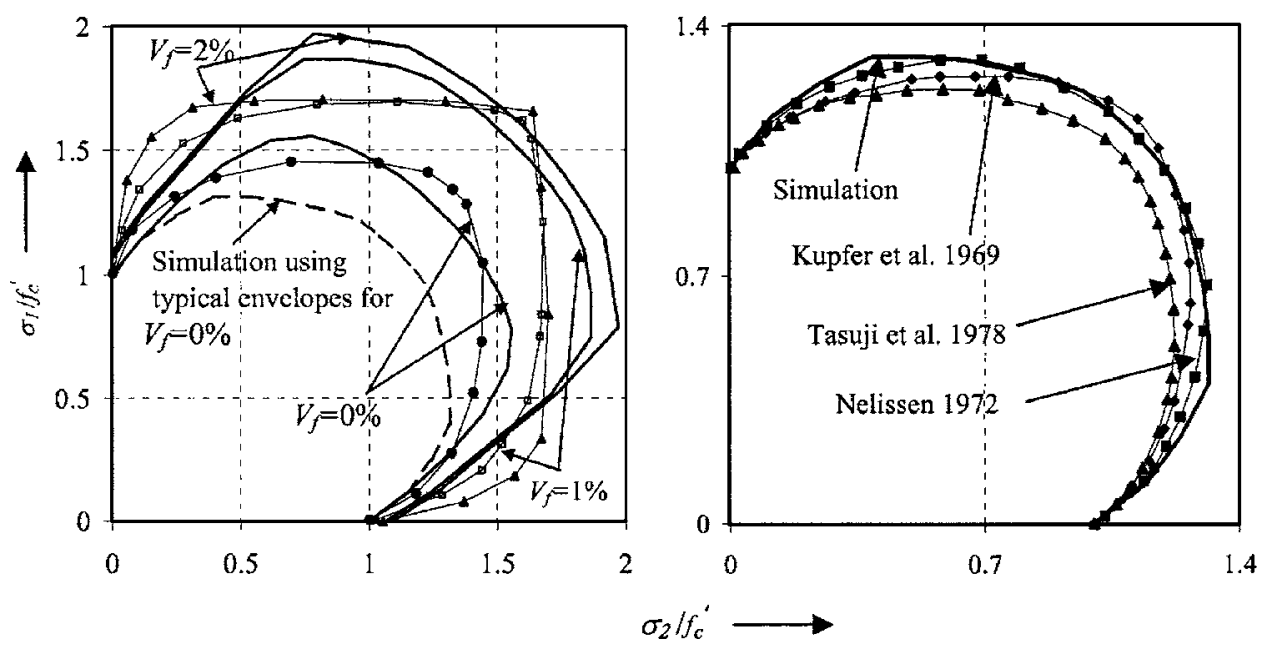

Fig. 6. Biaxial compression failure envelopes for fiber-reinforced concrete (left) and envelopes reported in literature for concrete (right) compared with numerical simulations

norm of $r_{i j}$ ). The solution of Eq. (9) and the algorithm for Model M5f follow similar steps to the algorithm for M5 (Bažant and Caner 2005b), which requires in each step computation of the fracturing tangential stiffness tensor from the tangential stiffnesses of the statically constrained microplanes.

The main differences from the M5 algorithm appear in the cohesive softening law part [substeps (i) through (v) of step (c) of the algorithm in the last section of Bažant and Caner (2005b)]. At the beginning of step (c) the following input values are available: $\gamma_{N}^{0}, \gamma_{M}^{0}$, and $\gamma_{L}^{0}$ (corresponding to the beginning of the loading step); the estimated current stress $\sigma_{N}, \sigma_{M}, \sigma_{L}$ (evaluated for the end of the current loading step); the estimated stress increments $\Delta \sigma_{N}, \Delta \sigma_{M}, \Delta \sigma_{L}$; and the maximum strain $\gamma_{u}^{0}$ attained in the previous loading history. Consequently, one can calculate $\quad \sigma=\left[\sigma_{N}^{2}+\left(\sigma_{M}^{2}+\sigma_{L}^{2}\right)\right]^{1 / 2}, \quad \Delta \sigma=\left[\Delta \sigma_{N}^{2}+\left(\Delta \sigma_{M}^{2}+\Delta \sigma_{L}^{2}\right)\right]^{1 / 2}$, $\gamma^{0}=\left\{\left(\gamma_{N}^{0}\right)^{2}+\left[\left(\gamma_{M}^{0}\right)^{2}+\left(\gamma_{L}^{0}\right)^{2}\right]\right\}^{1 / 2}$, and $\sigma^{b}$ (cohesive stress for FRC at $\left.\gamma^{0}\right)$.

Differently from the M5 algorithm [step (iv) in Bažant and Caner (2005b)], the strain for the case of growing fracture $\left(\Delta \sigma^{*}=\sigma-\sigma^{b}>0\right)$ is here updated according to

$$
\gamma=\gamma_{0}-\alpha\left(\mathrm{d} \sigma^{b} / \mathrm{d} \gamma\right)^{-1} \Delta \sigma^{*}
$$

where $\alpha=$ parameter in the range of $2-5$, used to accelerate convergence (for the present calculations, $\alpha=5$ ); and $\mathrm{d} \sigma^{b} / \mathrm{d} \gamma=$ derivative of the cohesive law (for concrete alone) at $\gamma=\gamma^{0}$; see Eq. (3). The fracturing compliances are given by

$$
\begin{gathered}
C_{N}=\left(\mathrm{d} \sigma^{b} / \mathrm{d} \gamma\right)^{-1} \gamma_{N}^{0} / \gamma^{0} \quad C_{M}=\left(\mathrm{d} \sigma^{b} / \mathrm{d} \gamma\right)^{-1} \gamma_{M}^{0} / \gamma^{0} \\
C_{L}=\left(\mathrm{d} \sigma^{b} / \mathrm{d} \gamma\right)^{-1} \gamma_{L}^{0} / \gamma^{0}
\end{gathered}
$$

In cases of unloading and reloading, $\sigma_{u}^{0}$ is computed from $\gamma_{u}^{0}$ using Eqs. (3) and (4). Then, in view of Eq. (5)

$$
\begin{gathered}
\gamma=\gamma_{u}^{0}+C_{u}(\sigma) \gamma_{u}^{0} \Delta \sigma^{*} / \sigma_{u}^{0} \\
C_{N}=C_{u}(\sigma) \gamma_{N}^{0} / \gamma^{0} \quad C_{M}=C_{u}(\sigma) \gamma_{M}^{0} / \gamma^{0} \quad C_{L}=C_{u}(\sigma) \gamma_{L}^{0} / \gamma^{0}
\end{gathered}
$$

The numerical algorithm converges fast, even for a large loading step (such as a strain increment of $10^{-5}$ ). However, one must be careful about the selection of element size $h$ controlling the softening band width in the fracturing zone, and the corresponding values of the softening cohesive law parameters (Bažant and Caner 2005a,b).

\section{Calibration, Numerical Results, and Data Fits}

Figs. 3(a-f), 4(a-f), and 6 compare the available experimental results (square, circular, triangular, or rhomboidal points) with the numerical fits achieved (continuous lines). Each diagram in the figures is plotted in terms of the average stress and average strain in the test specimen. All the simulations used the 37-point Gaussian integration formula for a unit hemisphere (Bažant and Oh 1986), which integrates exactly a polynomial of the 13th degree.

Figs. 3(a-c) show the data and fits for uniaxial tensile tests conducted by Li et al. (1998) on concretes with $V_{f}=0,2,3$, and $6 \%$. Three different types of reinforcement were used: (1) steel fibers "Dramix" (diameter $0.5 \mathrm{~mm}$, length $30 \mathrm{~mm}$, and hooked ends); (2) steel fibers "Harex" (having an arched cross section of dimensions $2.2 \times 0.25 \mathrm{~mm}$ and the length of $32 \mathrm{~mm}$ ); (3) synthetic fibers made of polyvinyl alcohol (PVA) (diameter $0.66 \mathrm{~mm}$, length $30 \mathrm{~mm}$ ).

Figs. 3(d-f), show the data and fits for the uniaxial tensile tests conducted by $\mathrm{Li}$ and $\mathrm{Li}$ (2001) on concrete reinforced by Dramix fibers, with three tests for $V_{f}=6 \%$, two for $7 \%$, and two for $8 \%$.

The optimal fit for these uniaxial tension tests is obtained by adjusting mainly the parameters of the cohesive softening law in Eqs. (3) and (4). The statically constrained microplane system is the only one active for this loading condition in the nonlinear range of material response. The parameters are identified starting with the concrete matrix, which is characterized by $E, \nu$, the adjustable parameters of Model M4, and the parameters of the cohesive law (3). For the diagrams in Figs. 3(a-c), $E=37 \mathrm{GPa}$, $\nu=0.18, k_{1}=0.000165, k_{2}=160, k_{3}=10, k_{4}=150, f_{t}^{\prime}=3.42 \mathrm{MPa}$, $p_{0}=-150, \quad \bar{\sigma}_{N}=2.55 \mathrm{MPa}, \bar{\gamma}_{N}=0.002$. For the diagrams in Figs. 3(d-f), the parameters differing from the foregoing ones are: $E=35 \mathrm{GPa}, p_{0}=-200, \bar{\gamma}_{N}=0.001$. The fiber effect is subsequently calibrated by adjusting parameters $p_{1}, p_{2}$, and $\sigma_{f}^{0}$ in Eq. (4) on the basis of the location of the hardening curve peaks. The optimized values are given in Table 1. 
Table 1. Parameters Used for the Fiber Cohesive Contribution in Uniaxial Tension Simulation

\begin{tabular}{lcccccccccccc}
\hline Fig. & $3(\mathrm{a})$ & $3(\mathrm{a})$ & $3(\mathrm{a})$ & $3(\mathrm{~b})$ & $3(\mathrm{~b})$ & $3(\mathrm{~b})$ & $3(\mathrm{c})$ & $3(\mathrm{c})$ & $3(\mathrm{c})$ & $3(\mathrm{~d})$ & $3(\mathrm{e})$ & $3(\mathrm{f})$ \\
\hline$V_{f}$ & $2 \%$ & $3 \%$ & $6 \%$ & $2 \%$ & $3 \%$ & $6 \%$ & $2 \%$ & $3 \%$ & $6 \%$ & $6 \%$ & $7 \%$ & $8 \%$ \\
$\sigma_{f}^{0}$ & 0.6 & 1.75 & 2.5 & 0.6 & 0.67 & 1.3 & 0.6 & 2.0 & 2.5 & 2.5 & 3.0 & 3.0 \\
$p_{1}$ & 250 & 250 & 290 & 100 & 150 & 170 & 250 & 250 & 350 & 350 & 360 & 370 \\
$P_{2}$ & 30 & 27 & 22 & 35 & 20 & 16 & 30 & 25 & 15 & 15 & 15 & 15 \\
\hline
\end{tabular}

In the case of high resistance fibers (such as Dramix 6,7,8\%, and Harex 6\%), the normal tensile stress in the kinematically constrained microplane system reaches the boundary surface during the initial elastic loading. Therefore, parameters $p_{3}$ and $p_{4}$ need to be calibrated in Eq. (8) to avoid spurious inelastic phenomena; their values for our computations are constant and equal to 20,000 and 1,000 , respectively. Besides, at large volume fraction of fibers, the measured stress-strain diagram of FRC exhibits a horizontal plateau [evident in Figs. 3(a and c) for $V_{f}=6 \%$ ]. This can be captured by calibrating the parameter $p_{s}$ as $p_{s}=0.05$ in Fig. 3(a) $\left(V_{f}=6 \%\right)$ and $p_{s}=0.1$ in Fig. 3(c) $\left(V_{f}=6 \%\right)$.

The experimental data in Figs. 4(a-f) were obtained by Chern et al. (1992) on fiber-reinforced concretes with carbon steel fibers of $V_{f}=0,1$, and $2 \%$. The specimens were loaded in multiaxial compression. Therefore, the response depends mainly on the kinematically constrained system. Here the properties of the concrete matrix are: $E=20 \mathrm{GPa}, \nu=0.18, k_{1}=0.000125, k_{2}=180$, $k_{3}=13, \quad k_{4}=70, \quad f_{t}^{\prime}=3.42 \mathrm{MPa}, \quad p_{0}=-150, \quad \bar{\sigma}_{N}=1.028 \mathrm{MPa}$, $\bar{\gamma}_{N}=0.00125$. The simulations of uniaxial compression shown in Fig. 4(a) are used to calibrate parameters $p_{5}$ and $p_{6}$ in Eq. (7). For hydrostatic compression [Fig. 4(b)], FRC exhibits no significant differences from normal concrete (at least for $V_{f} \leq 2 \%$ ). The triaxial compression tests in Figs. 4(c and d) are used to calibrate parameter $k_{2}$ since the failure mode involves shear [see Fig. 5(d) on the right]. The optimized values for $V_{f}=0,1$, and $2 \%$ are $p_{5}=0,3,580,4,000, p_{6}=0,235,200$, and $k_{2}=180,370$, and 1,050 , respectively.

Fig. 6 (left) shows the test results for biaxial compression of FRC with $V_{f}=0,1$, and $2 \%$ as reported in Yin et al. (1989). The concrete matrix in the numerical simulations is characterized by: $E=27 \mathrm{GPa}, \nu=0.18, k_{1}=0.000145, k_{2}=1,000, k_{3}=10, k_{4}=150$, $f_{t}^{\prime}=3.42 \mathrm{MPa}, p_{0}=-150, \bar{\sigma}_{N}=1.028 \mathrm{MPa}, \bar{\gamma}_{N}=0.00125$, which gives the compressive strength of $f_{c}^{\prime}=37.52 \mathrm{MPa}$. The optimized values for $V_{f}=0,1$, and $2 \%$ are $p_{5}=0,1,500,1,800$, and $p_{6}=0$, 200, 200, respectively. These parameters have been identified to match the mean value of the overall failure envelope. Discrepancies nevertheless appear.

Finally, Figs. 4(e and f) show a fit of a hydrostatic compression test followed by unloading. The simulations for both axial and transverse deformations are seen to closely follow the test results.

The foregoing simulations generally attain good agreement with experiments. This confirms the soundness of the present model. Discrepancies are nevertheless observed in Figs. 3(d-f), 4(c), and 6(a). In Figs. 3(d-f), the simulation yields a peak load followed by softening and rehardening, whereas the tests show a smooth hardening curve. This discrepancy is due to the inability of the cohesive curve to switch, for the given concrete matrix, from softening-hardening to pure hardening. In Fig. 4(c), the simulation of triaxial tests at low confinement pressure indicates a lower asymptotic stress than the tests. However, the relative changes of the asymptotic stress from one fiber volume fraction are correct. In Fig. 6 (left), the biaxial compression failure envelope shows discrepancies with the experiments, which can prob- ably be attributed to an atypical form of the experimental failure envelope for this concrete matrix, differing from most other concretes in the literature [see in Fig. 6 on the right the results by Kupfer et al. (1969), Tasuji et al. (1978), and Nelissen (1972)]. If these typical envelopes were used, the simulation would give a much better fit (continuous line in Fig. 6 on the right). This same simulation is reported for comparison in Fig. 6 on the left as dashed line.

Addition of several new parameters to those already involved in Models M4 and M5 might have been suspected of making Model M5f too difficult to calibrate. Not so, however, because the model parameters need not be optimized by simultaneous fitting of all the test data, and because the new parameters in M5f have a clear and easily identifiable trend with respect to $V_{f}$. The following sequential calibration procedure, in which only a few parameters are optimized to fit only one simple type of test, greatly simplifies the task.

1. Identify the parameters of Model M4 on the basis of concrete without fibers.

2. From the uniaxial tension data, identify $p_{1}, p_{2}$, and $\sigma_{f}^{0}$ (and, if needed, $p_{3}, p_{4}$, and $p_{s}$ ).

3. From the uniaxial compression data, identify $p_{5}$ and $p_{6}$.

4. From the triaxial compression data, identify $k_{2}$.

Task (1) is easy because most of the parameters that M5 shares with M4 can be kept fixed for all concretes, so that only four need to be calibrated. The calibration of these four can also be made sequentially, with simultaneous optimization of no more than two parameters [for details see Caner and Bažant (2000) and Bažant and Caner (2005a,b)].

Another application of M5f is the prediction of stress-strain curve for $V_{f}$ values different from those tested experimentally. This is achieved by interpolating the values of model parameters identified from tests for several different $V_{f}$ values. By fitting these parameter values, one can identify simple functions of the type $p_{1}\left(V_{f}\right), p_{2}\left(V_{f}\right)$, etc., to be used in calculations for arbitrary $V_{f}$. Still another application is to predict approximate stress-strain curves for multiaxial loading when only uniaxial test data are available.

\section{Remarks on Mesh Size Sensitivity and Fiber Size Effect}

Postpeak strain softening causes spurious mesh sensitivity of finite element computations because the energy dissipation per unit area of failure surface depends on the chosen finite element size dictating the width of the band into which cracking localizes. A simple way to avoid it is to apply the crack band model (Bažant and Oh 1983b), which adjusts the energy dissipation according to the ratio of the element size $h$ and the characteristic crack band width $l$ that gives correct energy dissipation as obtained in fracture tests. The crack band model has already been successfully implemented in commercial code ATENA, and so has model M4 (Červenka et al. 2004). 
The crack band model may be applied in the same way as described for M5 by Bažant and Caner (2005b). Briefly, the model must ensure constancy of the energy dissipated per unit length of crack band, which equals $l$ times the sum of the areas $W_{f}$ under the postpeak portions of all microplane stress-strain curves; $l=$ material characteristic length $\approx$ size of the material volume for which the microplane model has been calibrated. If the finite element size $h \neq l$, and if localization is expected, the softening stress-strain boundaries on the microplanes need to be rescaled, to ensure that $W_{f}^{*} h=W_{f} l$ or $W_{f}^{*}=W_{f} l / h$. In other words, all the (static and kinematic) stress-strain boundaries contributing to the overall fracture energy must be rescaled by a horizontal affinity transformation in the ratio $r=l / h$ (however, too small a ratio $r$ must be avoided to prevent snapback).

A more convenient way, with no need for rescaling the microplane model, is to apply the equivalent localization element (Červenka et al. (2005).

The size effect on structure strength depends on the fibers only through the material softening and fracture properties (Bažant and Chen 1997; Bažant and Planas 1998; Bažant 2002, 2004; RILEM 2004). In FRC, however, there is also, on the material scale, the size effect of fiber length, caused by propagation of debonding cracks around a fiber during its pullout from the crack face. This problem is analogous, though on a smaller scale, to the pullout of steel bars from a concrete block, investigated experimentally and analytically by Bažant and Sener (1988), Bažant and Desmorat (1994), and Bažant et al. (1995). Although their analysis, in principle, also applies to fiber pullout, it is not important for current FRC because the range of fiber lengths and diameters is quite narrow, with the length varying from 30 to $50 \mathrm{~mm}$, and the diameter from 0.4 to $0.8 \mathrm{~mm}$.

\section{Conclusions}

1. Thanks to the vectorial nature of its constitutive law, the microplane model is particularly suited for capturing the oriented nature of cracks and the fiber effect on their opening. The fibers are active mainly in the direction normal to the opening cracks. The prevalent distinct orientation of fiber action, which is a salient feature of FRC, is impossible to simulate with the traditional constitutive models expressed in terms of tensors and their invariants.

2. An essential feature of M5f is the coupling of both kinematically and statically constrained microplane systems. The kinematic one is required to model the triaxial behavior, especially in compression and shear, and also the resistance of fibers to crack opening of many orientations in the prepeak and early postpeak deformation. The static one can simulate wide (far-postpeak) opening of cracks of one dominant orientation approximately normal to the direction of tension.

3. Wide crack opening is what activates the resistance of fibers. Their effect can be easily captured by modifying the softening stress-strain boundaries (or softening yield limits) for the normal stress components on the microplanes.

4. The material parameters can be identified from tests by a simple sequential procedure. Close representation of the experimentally observed behavior is achieved, not only for uniaxial tensile loading, but also for biaxial and triaxial loading, compressive as well as tensile.

\section{Acknowledgments}

Partial financial support under U.S. National Science Foundation Grants CMS-0301145 and CMS-0556323 to Northwestern University is gratefully acknowledged.

\section{References}

Batdorf, S. B., and Budianski, B. (1949). "A mathematical theory of plasticity based on the concept of slip." Technical Note No. 1871, National Advisory Committee for Aeronautics, Washington, D.C.

Bažant, Z. P. (1985). "Mechanics of fracture and progressive cracking in concrete structures." Fracture mechanics of concrete: Structural application and numerical calculation, G. C. Sih and A. DiTommaso, eds., Martinus Nijhoff, Dordrecht, The Netherlands, pp. 1-94.

Bažant, Z. P. (2002). Scaling of structural strength, Hermes Penton Science, London (and French translation with updates, Hermes, Paris, 2004).

Bažant, Z. P. (2004). "Scaling theory for quasibrittle structural failure." Proc. Natl. Acad. Sci. U.S.A., 101(37), 13400-13407.

Bažant, Z. P., et al. (2000b). "Large-strain generalization of microplane model for concrete and application.” J. Eng. Mech., 126(9), 971-980.

Bažant, Z. P., Caner, F. C., Carol, I., Adley, M. D., and Akers, S. A. (2000a). "Microplane model M4 for concrete: I. Formulation with work-conjugate deviatoric stress." J. Eng. Mech., 126(9), 944-953.

Bažant, Z. P., and Caner, F. C. (2005a). "Microplane model M5 with kinematic and static constraints for concrete fracture and anelasticity. I. Theory." J. Eng. Mech., 131(1), 31-40.

Bažant, Z. P., and Caner, F. C. (2005b). "Microplane model M5 with kinematic and static constraints for concrete fracture and anelasticity. II. Computation.” J. Eng. Mech., 131(1), 41-47.

Bažant, Z. P., and Chen, E.-P. (1997). "Scaling of structural failure." Appl. Mech. Rev., 50(10), 593-627.

Bažant, Z. P., and Desmorat, R. (1994). "Size effect in fiber or bar pullout with interface softening slip." J. Eng. Mech., 120(9), 1945-1962.

Bažant, Z. P., and Jirásek, M. (2002). "Nonlocal integral formulations of plasticity and damage: Survey of progress." J. Eng. Mech., 128(11), 1119-1149.

Bažant, Z. P., Li, Z., and Thoma, M. (1995). "Identification of stress-slip law for bar or fiber pullout by size effect tests." J. Eng. Mech., 121(5), 620-625.

Bažant, Z. P., and Oh, B.-H. (1983a). "Microplane model for fracture analysis of concrete structures." Proc., Symp. on the Interaction of Non-Nuclear Munitions with Structures, U.S. Air Force Academy, Colorado Springs, Colo., 49-53.

Bažant, Z. P., and Oh, B.-H. (1983b). "Crack band theory for fracture of concrete." Mater. Constr. (Paris), 16, 155-177.

Bažant, Z. P., and Oh, B.-H. (1985). "Microplane model for progressive fracture of concrete and rock." J. Eng. Mech., 111(4), 559-582.

Bažant, Z. P., and Oh, B.-H. (1986). "Efficient numerical integration on the surface of a sphere." Z. Angew. Math. Mech., 66(1), 37-49.

Bažant, Z. P., and Pijaudier-Cabot, G. (1989). "Measurement of characteristic length of nonlocal continuum." J. Eng. Mech., 115(4), 755-767.

Bažant, Z. P., and Planas, J. (1998). Fracture and size effect in concrete and other quasibrittle materials, CRC Press, Boca Raton, Fla.

Bažant, Z. P., and Prat, P. C. (1988). "Microplane model for brittle plastic material: I. Theory." J. Eng. Mech., 114(10), 1672-1688.

Bažant, Z. P., and Şener, S. (1988). "Size effect in pullout tests." ACI Mater. J., 85, 347-351.

Bažant, Z. P., Xiang, Y. and Prat, P. C. (1996). "Microplane model for concrete: I. Stress-strain boundaries and finite strain." J. Eng. Mech., 1224(3), 245-254

Bažant, Z. P., and Zi, G. (2003). "Microplane constitutive model for porous isotropic rock." Int. J. Numer. Analyt. Meth. Geomech., 27, 25-47. 
Brocca, M., and Bažant, Z. P. (2001). "Microplane finite element analysis of tube-squash test of concrete with shear angle up to $70^{\circ}$." Int. J. Numer. Methods Eng., 52, 1165-1188.

Brocca, M., Bažant, Z. P., and Daniel, I. M. (2001). "Microplane model for stiff foams and finite element analysis of sandwich failure by core indentation." Int. J. Solids Struct., 38, 8111-8132.

Caner, F. C., and Bažant, Z. P. (2000). "Microplane model M4 for concrete: II. Algorithm and calibration." J. Eng. Mech., 126(9), 954-961.

Camacho, G. T., and Ortiz, M. (1996). "Computational modeling of impact damage in brittle materials." Int. J. Solids Struct., 33(20-22), 2899-2938.

Carol, I., and Bažant, Z. P. (1997). "Damage and plasticity in microplane theory.” Int. J. Solids Struct., 34(29), 3807-3835.

Carol, I., Jirásek, M., and Bažant, Z. P. (2004). "A framework for microplane models at large strain, with application to hyperelasticity." Int. J. Solids Struct., 41, 511-557.

Červenka, J., Bažant, Z. P., and Wierer, M. (2005). "Equivalent localization element for crack band approach to mesh-sensitivity in microplane model." Int. J. Numer. Methods Eng., 62(5), 700-726.

Chern, J.-C., Yang, H.-J., and Chen, H.-W. (1992). "Behavior of steel fiber-reinforced concrete in multiaxial loading." ACI Mater. J., 89(1), $32-40$.

Cho, S. H., and Kim, Y. I. (2003). "Effects of steel fibers on short beams loaded in shear." ACI Struct. J., 100(6), 765-774.

Grimaldi, A., and Luciano, R. (2000). "Tensile stiffness and strength of fiber-reinforced concrete." J. Mech. Phys. Solids, 48(9), 1987-2008.

Kabele, P. (2004). "Linking scales in modeling of fracture in high performance fiber-reinforced cementitious composites." Fracture Mechanics of Concrete Structures, Proc., FraMCoS-5, 5th Int. Conf. on Fracture Mechanics of Concrete and Concrete Structures, Vail, Colo., Vol. 1, V. C. Li, K. Y. Leung, K. J. Willam, and S. L. Billington, eds., IA-FraMCoS, Evanston, Ill., pp. 71-80.

Kholmyansky, M. M. (2002). "Mechanical resistance of steel fiberreinforced concrete to axial load." J. Mater. Civ. Eng., 14(4), 311-319.

Kullaa, J. (1994). "Constitutive modeling of fiber-reinforced concrete under uniaxial tensile loading." Composites, 25(10), 935-944.

Kupfer, H., Hilsdorf, H. K., and Rüsch, H. (1969). "Behavior of concrete under biaxial stresses." ACI J. 66(8), 656-666.

Kwak, Y. K., Eberhard, M. O., Kim, W. S., and Kim, J. (2002). "Shear strength of fiber-reinforced concrete beams without stirrups." $A C I$ Struct. J., 99(4), 530-538.

Li, F., and Li, Z. (2001). "Continuum damage mechanics based modeling of fiber-reinforced concrete in tension." Int. J. Solids Struct., 38, 777-793.

Li, Z., Li, F., Chang, T.-Y.-P, and Mai, Y.-W. (1998). "Uniaxial tensile behavior of concrete reinforced with randomly distributed short fibers." ACI Mater. J., 95(5), 564-574.

Mirsayah, A. A., and Banthia, N. (2002). "Shear strength of steel fiberreinforced concrete." ACI Mater. J., 99(5), 473-479.

Nataraja, M. C., Dhang, N., and Gupta, A. P. (1999). "Stress-strain curves for steel-fiber reinforced concrete under compression." Cem. Concr. Compos., 21(5-6), 383-390.

Nelissen, L. J. M. (1972). "Biaxial testing of normal concrete." Heron, 18(1), 1-90.

Pantazopoulou, S. J., and Zanganeh, M. (2001). "Triaxial tests of fiberreinforced concrete." J. Mater. Civ. Eng., 13(5), 340-348.

Peng, X., and Meyer, C. (2000). "A continuum damage mechanics model for concrete reinforced with randomly distributed short fibers." Comput. Struct., 78, 505-515.

Pizzari, G. A., Cangiano, S., and Cere, N. (2000). "Postpeak behavior of fiber-reinforced concrete under cyclic tensile loads." ACI Mater. J., 97(2), 182-192.

Ramesh, K., Seshu, D. R., and Prabhakar, M. (2003). "Constitutive behavior of fibre reinforced concrete under axial compression." Cem. Concr. Compos., 25(3), 343-350.

Reinhardt, H. W., and Cornelissen, H. A. W. (1984). "Post-peak cyclic behavior of concrete in uniaxial tensile and alternating tensile and compressive loading." Cem. Concr. Res., 14(2), 263-270.

RILEM Committee QFS. (2004). "Quasibrittle fracture scaling and size effect." Mater. Struct., 37(272), 547-586.

Soroushian, P., and Bayasi, Z. (1991). "Fiber-type effects on the performance of steel fiber-reinforced concrete." ACI Mater. J., 88(2), $129-134$.

Stroud, A. H. (1971). Approximate calculation of multiple integrals, Prentice-Hall, Englewood Cliffs, N.J.

Tasuji, M. E., Slate, F. O., and Nilson, A. H. (1978). "Stress-strain response and fracture of concrete in biaxial loading." ACI J., 75(7), 306-312.

Taylor, G. I. (1938). "Plastic strain in metals.” J. Inst. Met., 62, 307-324.

Traina, L. A., and Mansour, S. A. (1991). "Biaxial strength and deformational behavior of plain and steel fibre concrete." ACI Mater. J., 88(4), 354-362.

Yin, W. S., Su, E. C. M., Mansur, M. A., and Hsu, T. T. C. (1989). "Biaxial tests of plain and fiber concrete." ACI Mater. J., 86(3), 236-243. 
Copyright of Journal of Engineering Mechanics is the property of American Society of Civil Engineers and its content may not be copied or emailed to multiple sites or posted to a listserv without the copyright holder's express written permission. However, users may print, download, or email articles for individual use. 